\title{
Cosmic background of gravitational waves from rotating neutron stars
}

\author{
T. Regimbau and J. A. de Freitas Pacheco
}

\begin{abstract}
Observatoire de la Côte d'Azur, BP 4229, 06304 Nice Cedex 4, France
e-mail: pacheco@obs-nice.fr

Received 25 April 2001 / Accepted 10 May 2001

Abstract. The extragalactic background of gravitational waves produced by tri-axial rotating neutron stars was calculated, under the assumption that the properties of the underlying pulsar population are the same of those of the galactic population, recently derived by Regimbau \& de Freitas Pacheco (2000). For an equatorial ellipticity of $\varepsilon=10^{-6}$, the equivalent density parameter due to gravitational waves has a maximum amplitude in the range $2 \times 10^{-11}-3 \times 10^{-9}$, around $0.9-1.5 \mathrm{kHz}$. The main factors affecting the theoretical predictions are discussed. This background is comparable to that produced by the "ring-down" emission from distorted black holes. The detection possibility of this background by a future generation of gravitational antennas is also examined.
\end{abstract}

Key words. pulsars: general - gravitational waves - stars: neutron

\section{Introduction}

In the past years, a large number of papers devoted to stochastic backgrounds of gravitational waves appeared in the literature (see Maggiore 2000 for a recent review). Besides processes that took place very shortly after the big-bang, the emission from a large number of unresolved sources can produce a stochastic background. Supernovas (Blair et al. 1997) and distorted black holes (Ferrari et al. 1999a; de Araújo et al. 2000) are examples of sources able to generate shot noise, while a truly continuous background could be produced, for instance, by the " $r$-mode" emission from young and hot neutron stars (Owen et al. 1998; Ferrari et al. 1999b). Detection of such backgrounds may probe the cosmic star formation rate up to redshifts of $z \sim 4-5$, the mass range of the progenitors of neutron stars and black holes as well as the initial angular momentum of these objects.

The contribution of the entire population of rotating neutron stars to the continuous galactic background of gravitational waves was considered by different authors (Schutz 1991; Giazotto et al. 1997; de Freitas Pacheco \& Horvath 1997) and, more recently, this subject was revisited by Regimbau \& de Freitas Pacheco (2000, hereafter RP00). In the latter, the "true" population of rotating neutron stars was synthesized by Monte Carlo techniques and its contribution to the galactic background of gravitational waves was estimated. If the planned sensitivity of the first generation of laser beam interferometers is taken into account (in particular that of the French-Italian

Send offprint requests to: T. Regimbau, e-mail: regimbau@obs-nice.fr project VIRGO), then the simulations by RP00 suggest that only few objects will contribute to the signal, if the mean equatorial ellipticity of neutron stars is of the order of $\varepsilon=10^{-6}$. Upper limits on $\varepsilon$ have been obtained by assuming that the observed spin-down of pulsars is essentially due to the emission of gravitational waves. In this case, one obtains $\varepsilon \leq 10^{-3}$ for "normal" pulsars whereas recycled or rejuvenated pulsars seem to have equatorial deformations less than $10^{-8}$. Although the galactic population will not produce a real background, the integrated contribution of these objects remains to be investigated in a large volume of the universe. Estimates of this emission are important because it may rival with the background of possible cosmological origin. Since there is an upper limit to the wave frequency of the pulsar gravitational radiation, a putative cosmic background will dominate at low frequencies and the knowledge of the spectral energy distribution of the background produced by discrete sources may help in the choice of the best frequency domain to search for a relic emission.

In the present paper, the integrated gravitational emission of rotating neutron stars to the background is calculated under the assumption that the distributions of the rotation period and magnetic field derived by RP00 can conveniently be scaled to other galaxies. The plan of this paper is the following: in Sect. 2 the model computations are described; in Sect. 3 the results are discussed and finally, in Sect. 4 the conclusions are given.

\section{The model}

The main working hypothesis of our computations concerns the true rotation period and magnetic field 
distributions of pulsars. For galactic objects, these distributions were derived by RP00 using Monte Carlo simulations to reproduce the different observed distributions of physical parameters, like the period and its first derivative as well as distances, when selection effects are taken into account. These simulations permitted us to establish the parameters of the initial distribution of periods and magnetic fields. In the absence of similar results for the extragalactic pulsar population, we assumed here that pulsars are born everywhere with rotation periods and fields obeying those distribution laws.

For a single pulsar, the frequency distribution of the total emitted gravitational energy in the source's frame is

$$
\frac{\mathrm{d} E}{\mathrm{~d} \nu}=\frac{\mathrm{d} E}{\mathrm{~d} t}\left|\frac{\mathrm{d} t}{\mathrm{~d} \nu}\right|
$$

It is worth mentioning that in spite of $(\mathrm{d} E / \mathrm{d} t)$ in the above equation being the pulsar gravitational wave emission rate, the time variation of the frequency is fixed by the magnetic dipole emission, responsible for the deceleration of the star. This means that angular momentum losses by gravitational waves will never overcome those produced by magnetic torques, which is equivalent to say that the average equatorial deformation is always less than $10^{-3}$. Under these conditions, the energy frequency distribution, taking into account that the gravitational wave frequency is twice the rotational frequency is

$$
\frac{\mathrm{d} E}{\mathrm{~d} \nu}=\frac{256 G \pi^{6}}{5 c^{5}} \varepsilon^{2} I^{2}\left(\frac{\tau_{\mathrm{m}}}{P_{\mathrm{o}}^{2}}\right) \nu^{3}=K \nu^{3}
$$

where $I$ is the moment of inertia of the star, $\tau_{\mathrm{m}}$ is the magnetic braking timescale (see RP00), $P_{\mathrm{o}}$ is the initial period of the pulsar and the other symbols have their usual meaning.

In order to estimate the average ratio $\left(\tau_{\mathrm{m}} / P_{\mathrm{o}}^{2}\right)$, we adopted the following procedure. We have performed Monte Carlo simulations in which the distribution probabilities of the variables $\tau_{\mathrm{m}}$ and $P_{\mathrm{o}}$ are the same as RP00. The resulting distribution of the quantity $\log \left(\tau_{\mathrm{m}} / P_{\mathrm{o}}^{2}\right)$ is given in Fig. 1, and it can be fitted by a Gaussian with a mean equal to $<\log \left(\tau_{\mathrm{m}} / P_{\mathrm{o}}^{2}\right)>=12.544$. Adopting this value as representative of the whole population, the constant $K$ in Eq. (2) is $K=9.27 \times 10^{35}\left(\varepsilon_{-6}\right)^{2} \mathrm{erg} \mathrm{Hz}^{-4}$, where we have introduced the notation $\varepsilon_{-6}=\left(\varepsilon / 10^{-6}\right)$. The gravitational wave flux at frequency $\nu_{\mathrm{o}}$ (observer's frame) due to sources localized in the redshift shell $z$, $z+\mathrm{d} z$ is

$\mathrm{d} F_{\nu_{\mathrm{o}}}=\frac{1}{4 \pi d_{\mathrm{L}}^{2}} \frac{\mathrm{d} E}{\mathrm{~d} \nu}\left|\frac{\mathrm{d} \nu}{\mathrm{d} \nu_{\mathrm{o}}}\right| \mathrm{d} R(z)$

where $d_{\mathrm{L}}=(1+z) r$ is the distance-luminosity, $r$ is the proper distance and the observer's frequency $\nu_{\mathrm{O}}$ is related to the frequency $\nu$ at the source by $\nu=(1+z) \nu_{\mathrm{o}}$. The event rate inside the shell $z, z+\mathrm{d} z$ is

$\mathrm{d} R(z)=\lambda_{\mathrm{p}} R_{\mathrm{c}}(z) \frac{\mathrm{d} V}{\mathrm{~d} z} \mathrm{~d} z$
In the above equation, $R_{\mathrm{c}}(z)$ is the "cosmic" star formation rate, $\lambda_{\mathrm{p}}=\int_{10}^{40} \xi(m) \mathrm{d} m$ is the mass fraction of formed stars in the range 10-40 $M_{\odot}$, supposed to be the mass range of the pulsar progenitors, with $\xi(m)$ being the initial mass function. For a Salpeter's law $\left(\xi(m) \propto m^{-2.35}\right)$, $\lambda_{\mathrm{p}}=4.84 \times 10^{-3} M_{\odot}^{-1}$. The element of the comoving volume is

$\mathrm{d} V=4 \pi r^{2} \frac{c}{H_{\mathrm{o}}} \frac{\mathrm{d} z}{E\left(\Omega_{i}, z\right)}$

where $H_{\mathrm{o}}$ is the Hubble parameter and the function $E\left(\Omega_{i}, z\right)$ is defined by the equation

$E\left(\Omega_{i}, z\right)=\left[(1+z)^{2}\left(1+z \Omega_{\mathrm{m}}\right)-z(2+z) \Omega_{\mathrm{v}}\right]^{1 / 2}$

where $\Omega_{\mathrm{m}}$ and $\Omega_{\mathrm{v}}$ are respectively the density parameters due to matter (baryonic and non-baryonic) and the vacuum, corresponding to a non-zero cosmological constant. The equivalent density parameter due to the spatial curvature satisfies $\Omega_{k}=1-\Omega_{\mathrm{m}}-\Omega_{\mathrm{v}}$.

Combining these equations, the expected gravitational wave flux at the frequency $\nu_{\mathrm{o}}$ is

$F_{\nu_{\mathrm{o}}}=K \nu_{\mathrm{o}}^{3} \lambda_{\mathrm{p}}\left(\frac{c}{H_{\mathrm{o}}}\right)\left(\varepsilon_{-6}\right)^{2} \int_{0}^{z_{\max }} \frac{(1+z)^{2} R_{\mathrm{c}}(z)}{E\left(\Omega_{i}, z\right)} \mathrm{d} z$.

In the literature an equivalent density parameter due to gravitational waves is often used to measure the strength of the background at a given frequency, defined by the equation

$\Omega_{\mathrm{GW}}=\left(\frac{8 \pi G}{3 H_{\mathrm{o}}^{2}}\right) \frac{\nu_{\mathrm{o}} F_{\nu_{\mathrm{o}}}}{c^{3}}$.

\subsection{Numerical calculations}

In order to evaluate numerically Eqs. (7)-(8), it is necessary to specify the cosmic star formation rate $R_{\mathrm{c}}(z)$ and the parameters of the world model, namely, the values of $H_{\mathrm{o}}, \Omega_{\mathrm{m}}$ and $\Omega_{\mathrm{v}}$.

Madau \& Pozzetti (1999) have reviewed the constraints imposed by the observed extragalactic background light on the cosmic star formation rate (CSFR). They concluded that after an extinction correction of $A_{1500}=1.2 \mathrm{mag}\left(A_{2800}=0.55 \mathrm{mag}\right)$, a star formation rate given by the relation

$R_{\mathrm{c}}(z)=\frac{0.23 \mathrm{e}^{3.4 z}}{\left(44.7+\mathrm{e}^{3.8 z}\right)} M_{\odot} \mathrm{yr}^{-1} \mathrm{Mpc}^{-3}$

fits all measurements of the UV-continuum and $\mathrm{H} \alpha$ luminosity densities well from the present epoch up to $z=4$. However, according to Hopkins et al. (2001), even when reddening corrections are taken into account, significant discrepancies still remain between the CSFR derived from $\mathrm{UV}-\mathrm{H} \alpha$ measurements and those derived from far-infrared and radio luminosities, which are not affected by dust extinction. Hopkins et al. (2001) assumed a reddening correction dependent on the star formation rate and obtained 


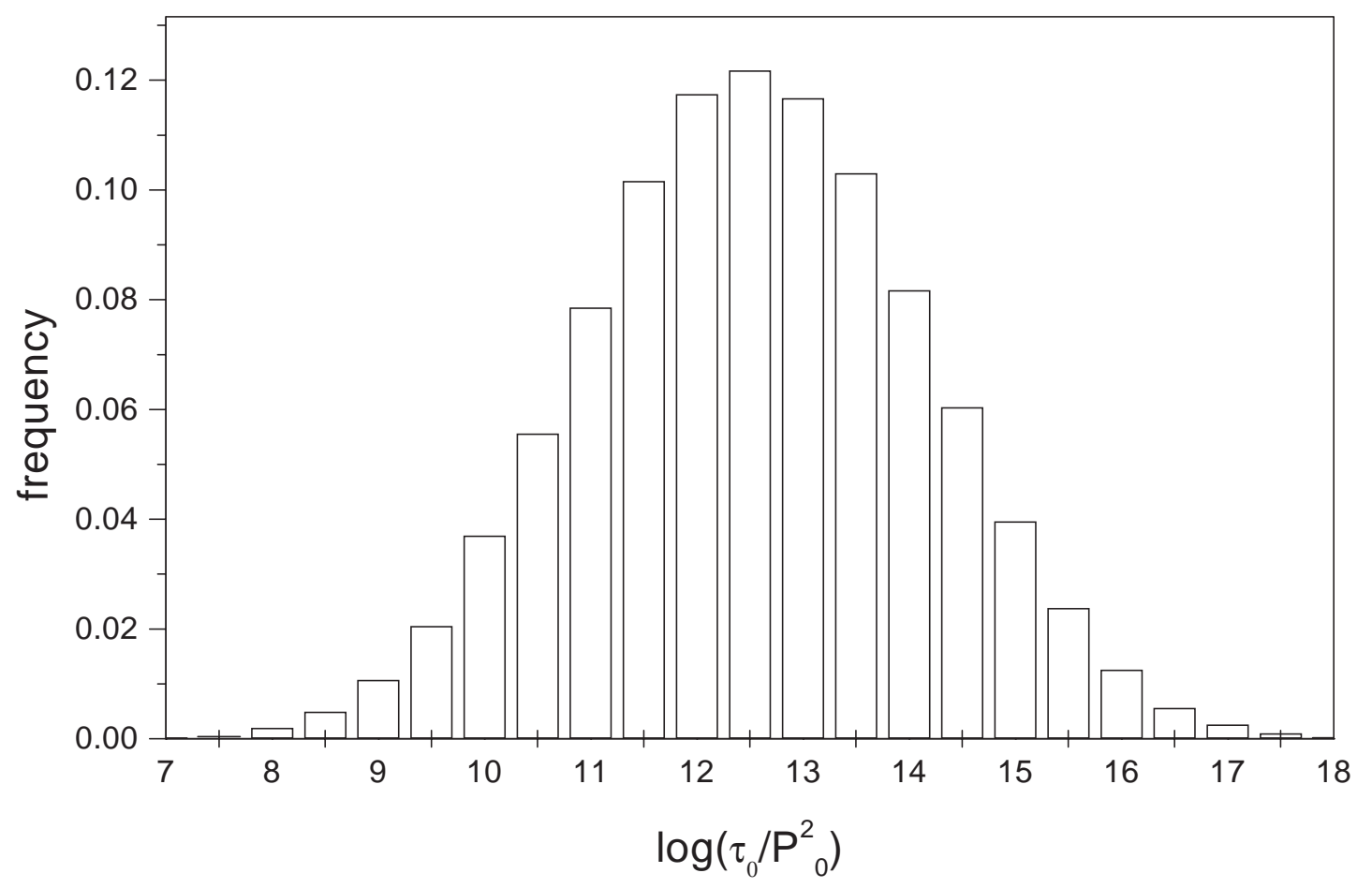

Fig. 1. Simulated distribution of the quantity $\log \left(\tau_{\mathrm{m}} / P_{\mathrm{o}}^{2}\right)$.

a good agreement between the CSFRs derived from different set of measurements. We have fitted their results by a function similar to Eq. (9), namely,

$R_{\mathrm{c}}(z)=\frac{1.207 \mathrm{e}^{3.836 z}}{\left(39.970+\mathrm{e}^{4.163 z}\right)} M_{\odot} \mathrm{yr}^{-1} \mathrm{Mpc}^{-3}$.

Taking into account the uncertainties still present in the derivation of the CSFR, we have performed calculations using both rates.

Recent BOOMERANG and MAXIMA results (de Bernardis et al. 2000; Hanany et al. 2000) on the power spectra of the cosmic microwave background and observations of distant type Ia supernovas (Perlmutter et al. 1999; Schmidt et al. 1998), which suggest that the expansion of the Universe is accelerating, support a spatially flat geometry and a non-zero cosmological constant. Both sets of data are consistent with $\Omega_{\mathrm{m}}=0.30$ (including baryonic and non-baryonic matter) and $\Omega_{\mathrm{v}}=0.70$, which will be adopted in our computations. However, no significant differences in our results were observed if a model defined by $\Omega_{\mathrm{m}}=1$ and $\Omega_{\mathrm{v}}=0$ is adopted. The Hubble parameter $H_{\mathrm{o}}$ was taken to be equal to $68 \mathrm{kms}^{-1} / \mathrm{Mpc}^{-1}$ (Krauss 2001).

RP00 assume in their simulations that the maximum rotation frequency of a newly born pulsar is $2000 \mathrm{~Hz}$, which corresponds to a gravitational wave frequency of $4000 \mathrm{~Hz}$. If the upper limit of the integral in Eq. (7) is $z_{\max }=5$, then the maximum frequency seen by the observer is $\approx 660 \mathrm{~Hz}$. For higher frequencies, only near objects will contribute to the integral and the upper limit should be replaced by $z_{\max }=\left(4000 / \nu_{\mathrm{o}}\right)-1$, with $\nu_{\mathrm{o}}$ in $\mathrm{Hz}$. This parameter affects the resulting spectrum, as we shall see below. Thus, calculations with a different cutoff were also performed.

\section{Results}

Figure 2 shows the density parameter $\Omega_{\mathrm{GW}}$ as a function of the frequency. Labels M1 and H1 correspond to star formation rates given respectively by Eqs. (9) and (10) and a maximum gravitational wave frequency equal to $4000 \mathrm{~Hz}$. The labels M2 and H2 have the same meaning but here the maximum gravitational wave frequency cutoff is at $2000 \mathrm{~Hz}$, corresponding to a minimum rotation period of $1 \mathrm{~ms}$. All these curves were calculated for an equatorial deformation $\varepsilon=10^{-6}$, and we recall that the results scale as $\varepsilon^{2}$. The numerical calculations indicate a broad maximum around $1.5 \mathrm{kHz}$, if the maximum possible pulsar rotation frequency is $2 \mathrm{kHz}$. Decreasing this limit by a half, the spectrum narrows and the maximum shifts toward lower frequencies $(\sim 0.9 \mathrm{kHz})$. The amplitude is also affected, being reduced by almost one order of magnitude. This happens because according to Eq. (8), the amplitude grows as $\nu_{\mathrm{o}}^{4}$ but a lower frequency cutoff implies that only nearby objects will contribute to the integrated signal, thus reducing the amplitude at maximum.

If the equatorial ellipticity may reach values of the order of $10^{-6}$, then the energy density of the background generated by pulsars may be comparable and even higher than that expected from newly born black holes (Ferrari et al. 1999a; de Araújo et al. 2000), originating from the collapse of massive stars $\left(M \geq 40 M_{\odot}\right)$. For a comparison, the spectrum corresponding to the ring-down emission from distorted black holes calculated by Ferrari et al. (1999a) is also plotted in Fig. 2, appropriately scaled to 


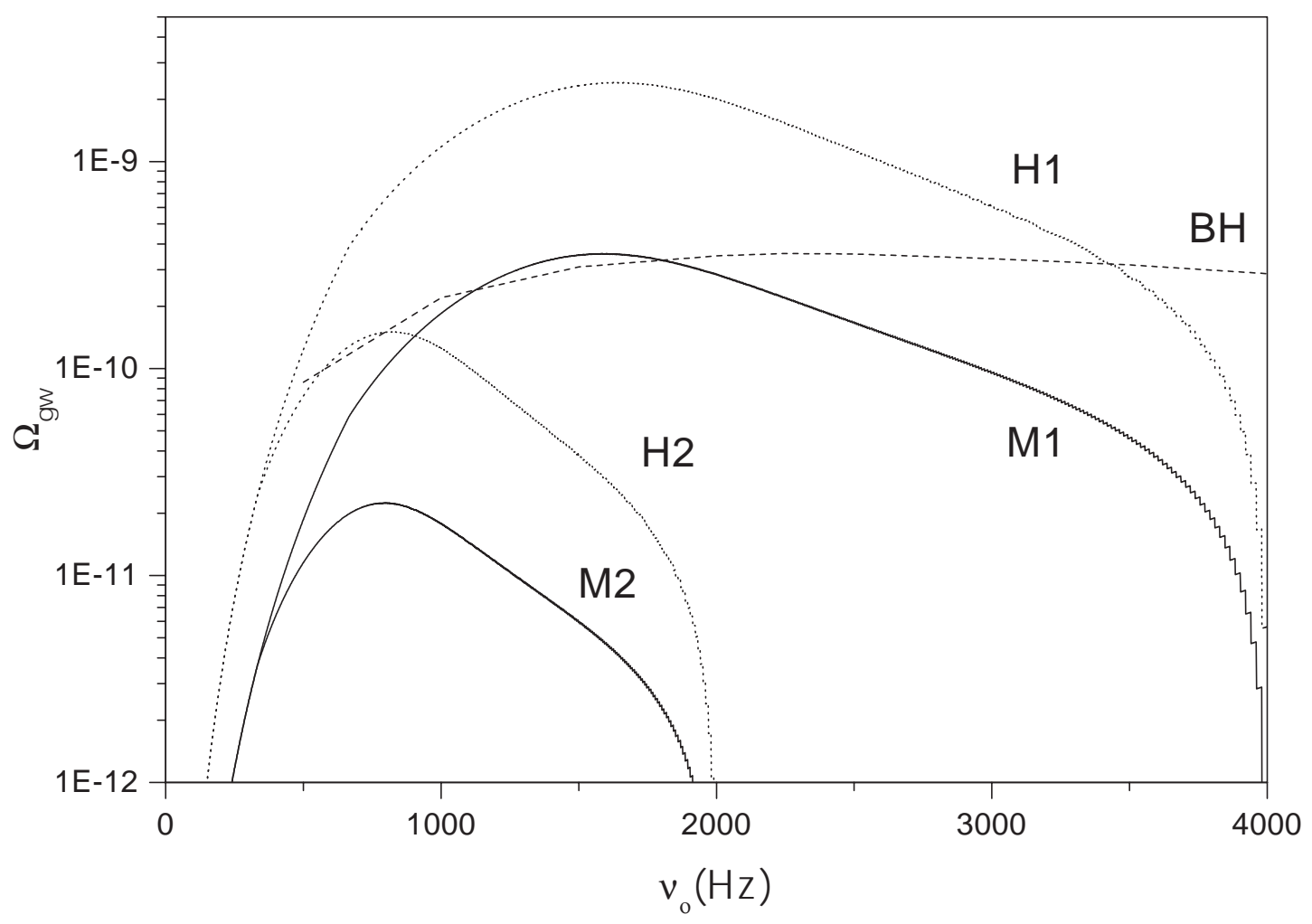

Fig. 2. Density parameter of GW versus frequency. Labels $M$ and $H$ correspond respectively to cosmic star formation rates given by Eqs. (9) and (10), whereas labels 1 and 2 correspond to maximum rotation frequencies of 2.0 and $1.0 \mathrm{kHz}$. The predicted background produced by distorted black holes (Ferrari et al. 1999a) is also shown for comparison.

the Hubble parameter here adopted. In the case of distorted black holes, the uncertainties on the estimates of the background energy density rest on the conversion efficiency of the mass energy into gravitational waves as well as on the minimum mass of the progenitor.

Hot and fast-rotating newly formed neutron stars may be unstable against the $r$-mode instability. Ferrari et al. (1999b) estimated that if all newly born neutron stars cross the "instability window" (see, for instance, Andersson et al. 2000), then the resulting density parameter has a maximum amplitude of $\Omega_{\mathrm{GW}} \approx 2 \times 10^{-8}$ in the frequency range $0.5-1.7 \mathrm{kHz}$. This signal would be by far the dominant component of the background at these frequencies. However, according to the simulations by RP00, only few pulsars are born within the instability window, reducing the amplitude of the background due to such a mechanism by orders of magnitude.

Unless the equatorial ellipticity of pulsars were substantially higher than the present expectations, the background generated by rotating neutron stars will hardly be detected by the present generation of laser beam interferometers and/or resonant detectors, but this could be a possibility for future projects presently under consideration, such as the Large Scale Cryogenic Gravitational Wave Telescope (LCGT), sponsored by the University of Tokyo and the European antenna EURO (W. Winkler, private communication). The former, with a baseline of $3 \mathrm{~km}$, is expected to have a $100 \mathrm{~W}$ laser and cooled sapphire mirrors among other technological improvements.
Therefore, one may expect that advanced laser beam interferometers may attain in the near future a sensitivity around $1 \mathrm{kHz}$, corresponding to a strain noise $\sqrt{S_{n}(\nu)}$ of about $10^{-25} \mathrm{~Hz}^{-1 / 2}$. On the other hand, the best strategy to detect the signal when the detector output is dominated by the noise, which is the present case, is to correlate data from two different gravitational antennas and to assume that they have independent noise. One interesting possibility would be to correlate the output of such an advanced detector with a resonant mass detector located at the same site, having a spherical or truncated icosahedron geometry. The advantages of this geometry with respect to a longitudinal bar is that a free elastic sphere has five degenerate quadrupole modes, each of which is sensitive to a different polarization and wave direction. Moreover, for a given material and resonant frequency, a spherical detector has a cross section larger than a cylindrical one. The sensitivity of resonant spheres is limited by Brownian motion noise associated with dissipation in the antenna and transducer, as well as by the electronic noise from amplifiers. In this case, the strain noise at resonance is approximately (Coccia \& Fafone 1997)

$\sqrt{S_{n}(\nu)}=\left(\frac{4 k T}{F_{n} Q_{n} M_{\mathrm{s}} v_{\mathrm{s}}^{2} \nu_{n}}\right)^{1 / 2}$

where $k$ is the Boltzmann constant, $T$ is the sphere temperature, $F_{n}$ is a dimensionless coefficient depending on each quadrupole mode $\left(F_{1}=2.98, F_{2}=1.14, F_{3}=0.107\right)$, $Q_{n}$ is the quality factor of the mode, $M_{\mathrm{S}}$ is the mass of 
the sphere, $v_{\mathrm{s}}$ is the velocity of the sound and $\nu_{n}$ is the mode frequency. For practical purposes, let us consider a sphere constituted of the aluminium alloy Al-5056. This material has a sound velocity of $5440 \mathrm{~ms}^{-1}$ and Coccia et al. (1996) have reported $Q$ values as high as $10^{8}$ for temperatures below $100 \mathrm{mK}$. A sphere with a diameter of $3.5 \mathrm{~m}$ (mass of 60.3 tons) has the two main frequencies of the quadrupole modes at $0.8 \mathrm{kHz}$ and $1.5 \mathrm{kHz}$, covering quite well the predicted interval where the maximum amplitude of the pulsar background should occur. Assuming a typical temperature of $20 \mathrm{mK}$, the expected strain noise derived from Eq. (11) is $\sqrt{S_{n}\left(\nu_{1}\right)} \approx 1.5 \times 10^{-24} \mathrm{~Hz}^{-1 / 2}$.

If $\Delta \nu \approx 20 \mathrm{~Hz}$ is the bandwidth of the resonant mass detector and $\tau$ is the integration time, then the expected optimized signal-to-noise $S / N$ of the correlated outputs is (Allen 1997)

$\frac{S}{N}=\frac{3 H_{\mathrm{o}}^{2}}{\sqrt{50} \pi^{2}} \sqrt{\Delta \nu \tau} \frac{\Omega_{\mathrm{GW}}}{\nu^{3} \sqrt{S_{1}(\nu) S_{2}(\nu)}}$.

For one year integration, one obtains from the equation above $S / N \approx 0.2$, indicating that new technology detectors may in the future reach the required sensitivity to detect such a signal.

\section{Conclusions}

The contribution of rotating neutron stars to the extragalactic background of gravitational waves was calculated, under the assumption that the parameters characterizing the galactic population of pulsars derived by RP00 are the same everywhere. The amplitude of the equivalent density parameter attains a maximum in the frequency interval $0.9-1.5 \mathrm{kHz}$ and is in the range $10^{-11}$ to $3 \times 10^{-9}$. The amplitude scales as $\varepsilon^{2}$ and, for a given equatorial ellipticity, the main uncertainties in the amplitude are essentially due to the cosmic star formation rate and to the rotation frequency limit at the pulsar birth, which depends on the equation of state of the nuclear matter. For "realistic" equations of state, these limits are in the rotation period range $0.5-1.0 \mathrm{~ms}$, values adopted in our calculations.

The present estimates indicate that this background, having a duty cycle (measured by the product of the typical duration of the signal and the mean birth frequency of pulsars) greater than one, may have an energy density comparable to that produced by "ring-down" black holes. This emission is unlikely to be detected by the present generation of detectors.
Correlated advanced detectors may reach a limit of about $\Omega_{\mathrm{GW}} \approx 10^{-10}$ for a flat spectrum (Maggiore 2000), which is not the present case. However, new technology detectors, which are presently under consideration, may attain the required sensitivity. In particular, taking into account the low cost of a resonant mass detector compared to that of a laser interferometer, the installation in the same site of a "sphere" operating near the maximum predict frequency $(\sim 0.9-1.5 \mathrm{kHz})$, could be an adequate strategy to detect such a signal in the future.

\section{References}

Allen, B. 1997, in Relativistic Gravitation and Gravitational Radiation, ed. J.-A. Marck, \& J.-P. Lasota (Cambridge University Press), 373

Andersson, N, Jones, D. I., Kokkotas, K. D., \& Stergioulas, N. 2000, ApJ, 534, 75

Blair, D. G., Burman, R., Woodings, L. J. S., Mulder, M., \& Zadnik, M. G. 1997, in Omnidirectional Gravitational Radiation Observatory, ed. W. F. Velloso, O. D. Aguiar, \& N. S. Magalhães (World Scientific), 251

Coccia, E., Fafone, V., Frossati, G., ter Haar, E., \& Meisel, M. W. 1996, PRLA, 219, 263

Coccia, E., \& Fafone, V. 1997, in Omnidirectional Gravitational Radiation Observatory, ed. W. F. Velloso, O. D. Aguiar, \& N. S. Magalhães (World Scientific), 113

de Araújo, J. C. N., Miranda, O. D., \& Aguiar, O. D. 2000, PRD 61, 124015

de Bernardis, P., et al. 2000, Nature, 404, 995

de Freitas Pacheco, J. A., \& Horvath, J. E. 1997, PRD, 56, 859

Ferrari, V., Matarrese, S., \& Schneider, R. 1999a, MNRAS, 303,247

Ferrari, V., Matarrese, S., \& Schneider, R. 1999b, MNRAS, 303,258

Giazotto, A., Bonazzola, S., \& Gourgoulhon, E. 1997, PRD, 55,2015

Hanany, S., et al. 2000, ApJL, 545, 5

Hopkins, A. M., Connoly, A. J., Haarsma, D. B., \& Cram, L. E. 2001 [astro-ph/0103253]

Krauss, L. M. 2001 [astro-ph/0102305]

Madau, P., \& Pozzetti, L. 1999, MNRAS, 312, 9

Maggiore, M. 2000, Phys. Rep., in press [gr-qc/9909001]

Owen, B. J., Lindblom, L., Cutler, C., et al. 1998, PRD, 58, 084020

Perlmutter, S., et al. 1999, ApJ, 517, 565

Regimbau, R., \& de Freitas Pacheco, J. A. 2000, A\&A (RP00)

Schmidt, B., et al. 1998, ApJ, 507, 46

Schutz, B. 1991, in The Detection of Gravitational Waves, ed. D. B. Blair (Cambridge University Press) 